\title{
The Classic
}

\section{The Bonding of Prostheses to Bone by Cement}

\author{
Sir John Charnley MD
}

\begin{abstract}
This Classic Article is a reprint of the original work by Sir John Charnley, The Bonding of Prostheses to Bone by Cement. An accompanying biographical sketch of Sir John Charnley, MD is available at DOI 10.1007/ s11999-010-1547-6. The Classic Article is reproduced and adapted with permission and copyright (C) of the British Editorial Society of Bone and Joint Surgery from Charnley J. The bonding of prostheses to bone by cement. J Bone Joint Surg [Br]. 1964;46-B:518-529.
\end{abstract}

(C) The Association of Bone and Joint Surgeons ( 2010

Richard A. Brand MD ( $\square)$

Clinical Orthopaedics and Related Research, 1600 Spruce Street, Philadelphia, PA 19103, USA

e-mail: dick.brand@clinorthop.org
Since the preliminary communication on the bonding of femoral head prostheses into the upper end of the femur by cold-curing acrylic cement (Charnley 1960) a total of 455 prostheses have now been inserted by this means.

This report on experience with cold-curing acrylic cement in contact with living human bone is concerned with 1) the frequency of loosening of the cement-bone bond, as tested directly during re-exploration of the prosthesis or at necropsy; 2) the macroscopic, microscopic and radiographic appearances of the cement-bone bond in specimens; 3) the correlation of radiographic appearances with the state of fixation or looseness where this had been observed directly; 4) the best way to use the cement.

\section{Necropsy Studies}

Six necropsy specimens were studied. Three were from patients who died of natural causes between one and a half and three and a quarter years after operation, who had undergone prosthetic replacement for fractures of the femoral neck. The clinical results had been classed as very good; the patients were seventy-two, eighty-one and eighty-five, and, though not very active during the last six months of their lives, had been fairly active before.

The fourth specimen was from a patient of fifty-seven with severe polyarthritis who died one and a half years after operation from gold nephropathy.

The remaining two specimens were from patients who died as a result of a second operation: one with ankylosing spondylitis who died of acute gastric dilation following spinal osteotomy five months after a successful "low friction" arthroplasty; the other, a woman of seventy-five with osteoarthritis, who died of myocardial failure after removal of the hip prosthesis which was necessary because of failure of the plastic acetabulum after three and a quarter years.

In all of these specimens the femoral head prostheses were found to be rigidly fixed in the femur. The specimens, including the metal prostheses, were bisected with a metalcutting saw and photographed. Histological preparations were made after dissolving the cement with chloroform.

In one specimen from a woman of seventy-two, which was typical of all six, a stainless steel Austin Moore prosthesis had been cemented in three and a quarter years before and a Teflon socket had been inserted as a 
low-friction acetabular prosthesis. Function had been excellent for the first three years but then pain had returned and weight bearing had become defective as a result of wearing out of the Teflon socket. The cement here did not contain a radio-opaque tracer and the radiograph of the specimen (Fig. 1), with detail unobscured by surrounding soft parts, showed no bone reaction to demarcate the limits

Fig. 1 Radiograph showing no demarcation between cement and cancellous bone. This prosthesis was tight after three and a quarter years.

Fig. 2 The limits of the cement.

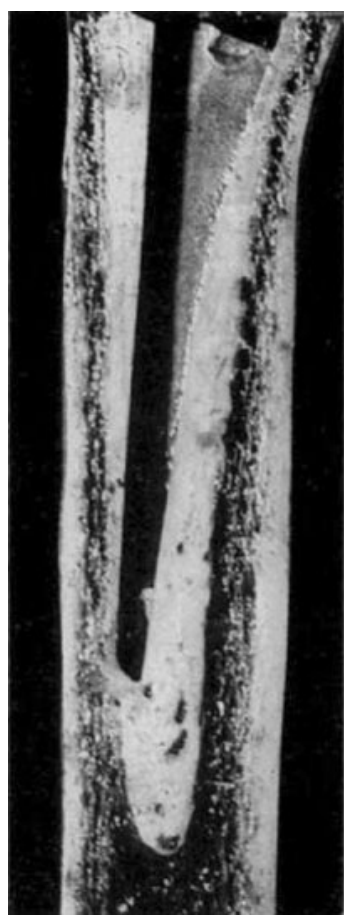

of the cement. Radiological evidence of demarcation between the cement and the cancellous bone was absent in all six specimens. It was only when the cut specimen was examined (Fig. 2) that the limits of the cement could be seen.

In five of the six specimens a fine white line of fibrous tissue between the surface of the cement and the cancellous bone could be seen. Without a lens this fibrous layer was hardly visible in the distal half of the specimen and was more obvious in the proximal part, where the cement lay in soft cancellous bone. The thickness of the fibrous layer was estimated at about 1 millimetre in the proximal part and about 0.25 millimetre or even less in the distal part. It is interesting that radiographically there was no suggestion of this line of fibrous tissue being demarcated by condensation in adjacent cancellous bone. In this respect the appearances are quite different from the radiographic appearances some time after insertion of an acrylic Judet prosthesis, when there is always a faint line of condensation in the layer of cancellous bone in contact with it (Fig. 3).

In one specimen the cement in the proximal part could be peeled away from the fibrous layer leaving a relatively smooth surface (Fig. 4) and the cement removed at this level had a nodular appearance (Fig. 5). The roughness of the surface of the cement is always an exact replica of the surface of the cancellous bone at the time of operation; and when cancellous bone has been curetted the surface of the cement is smoother. When this technique was being developed curettage was always used, but curettage is now

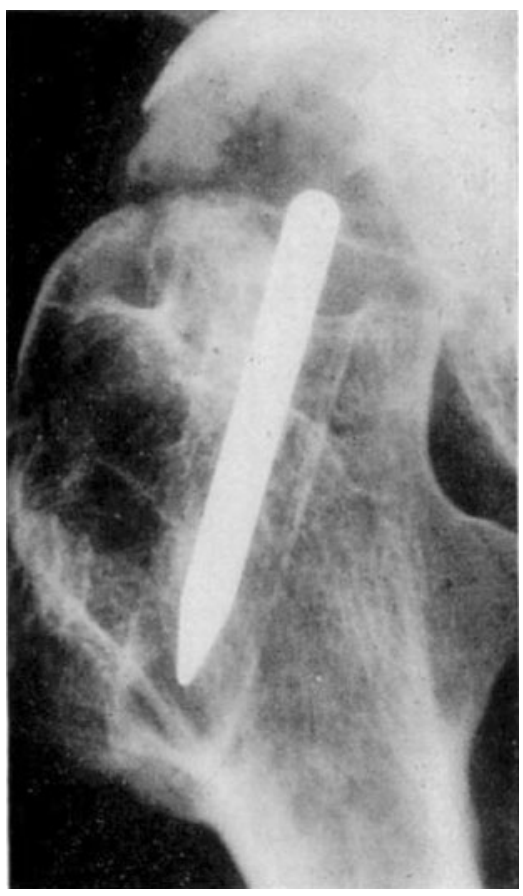

Fig. 3 Condensation in cancellous bone demarcating radiotranslucent plastic and bone. This condensation usually indicates motion between prosthesis and bone. 


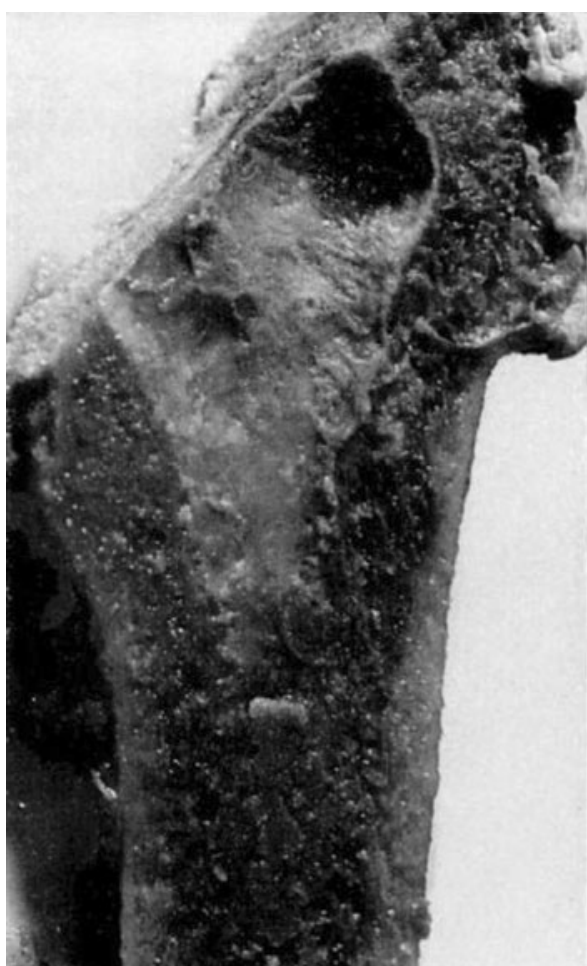

Fig. 4 Relatively smooth bed lined with fibrous tissue after extraction of cement. This bone had been curetted at time of insertion.

Fig. 5 Showing the relatively smooth surface of cement.

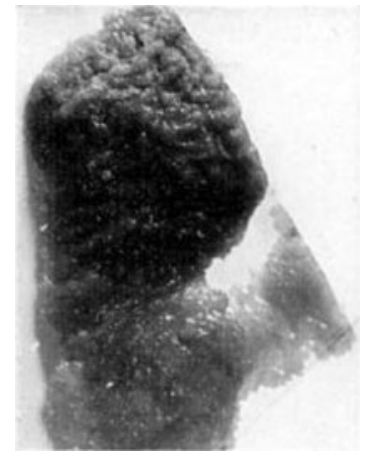

Fig. 6 Showing the rough surface from a specimen where curettage was not used.

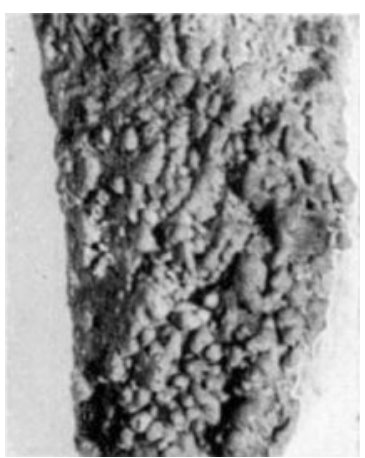

avoided in order to present the roughest possible surface to the cement (Fig. 6).

In one of the six specimens, a woman of eighty-one dying of natural causes one and a half years after

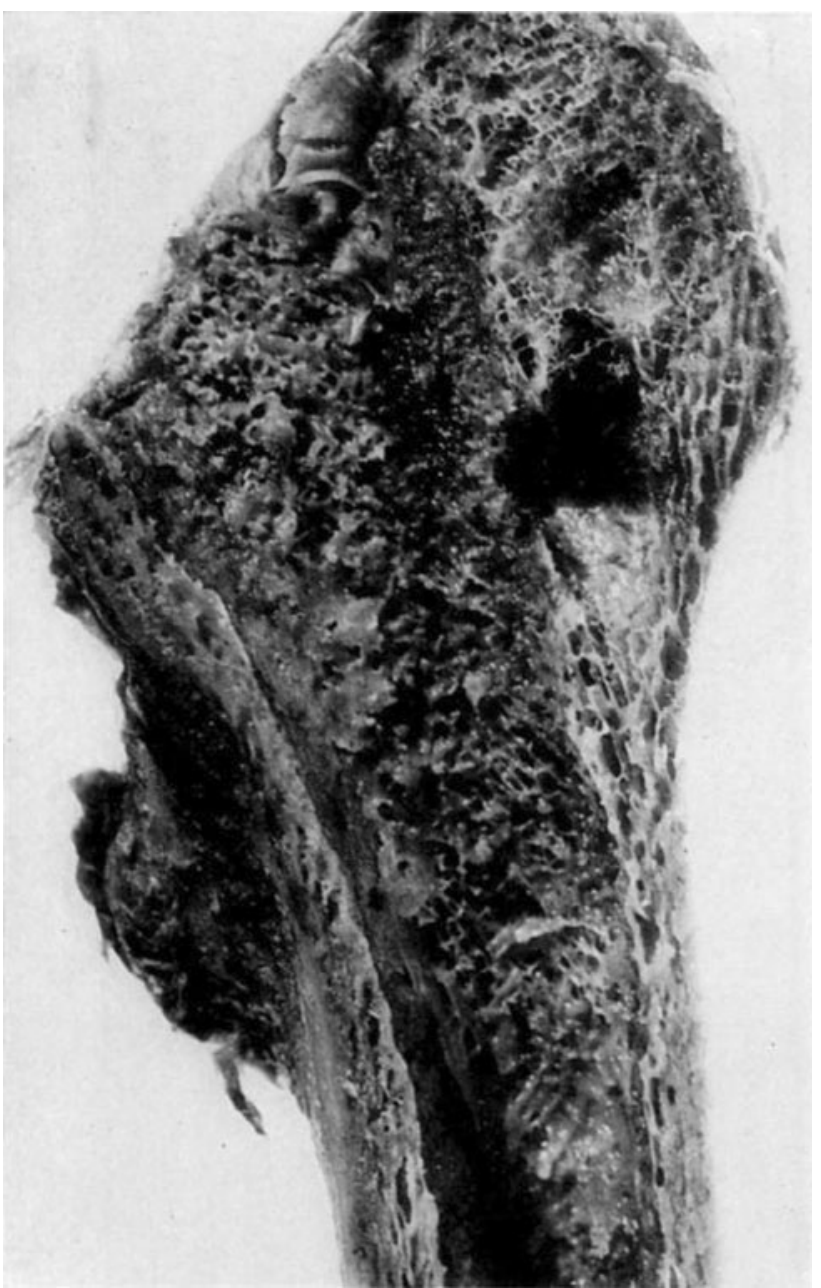

Fig. 7 The femur after dissolving away cement with chloroform. The prosthesis had been in situ one and a half years. Note the change of the inner layers of the cortex to cancellous structure without loosening of cement.

cementing an Austin Moore prosthesis, no naked-eye evidence of a fibrous intersection could be seen and this was demonstrated very effectively by dissolving away the cement with chloroform. This revealed the surface of the cancellous bone in contact with the cement unobscured by a thick layer of fibrous tissue (Fig. 7). The histology in this case revealed a fine layer of fibrous tissue about $5 \mu$ thick. This patient lived a normal active life, considering her age, for twelve of the eighteen months she lived after the operation.

These specimens reveal an interesting point in relation to osteoporosis of the cortical bone of the femoral shaft. As shown in Fig. 7 the inner layers of the cortex of the femoral shaft can revert to cancellous bone. The radiographic appearance of enlargement of the medullary canal suggests that the prosthesis may be loosening but direct examination shows that this appearance does not necessarily mean loosening as all the prostheses were held tight. 
Fig. 8 Section of cancellous bone in specimen illustrated in Fig. 7. A indicates site of cement. $\mathrm{B}$ is layer of fibrous tissue. $\mathrm{C}$ is dead cancellous bone. D is new lamellar bone deposited on dead bone and closing the trabecular space. E shows fatty marrow with normal appearance.

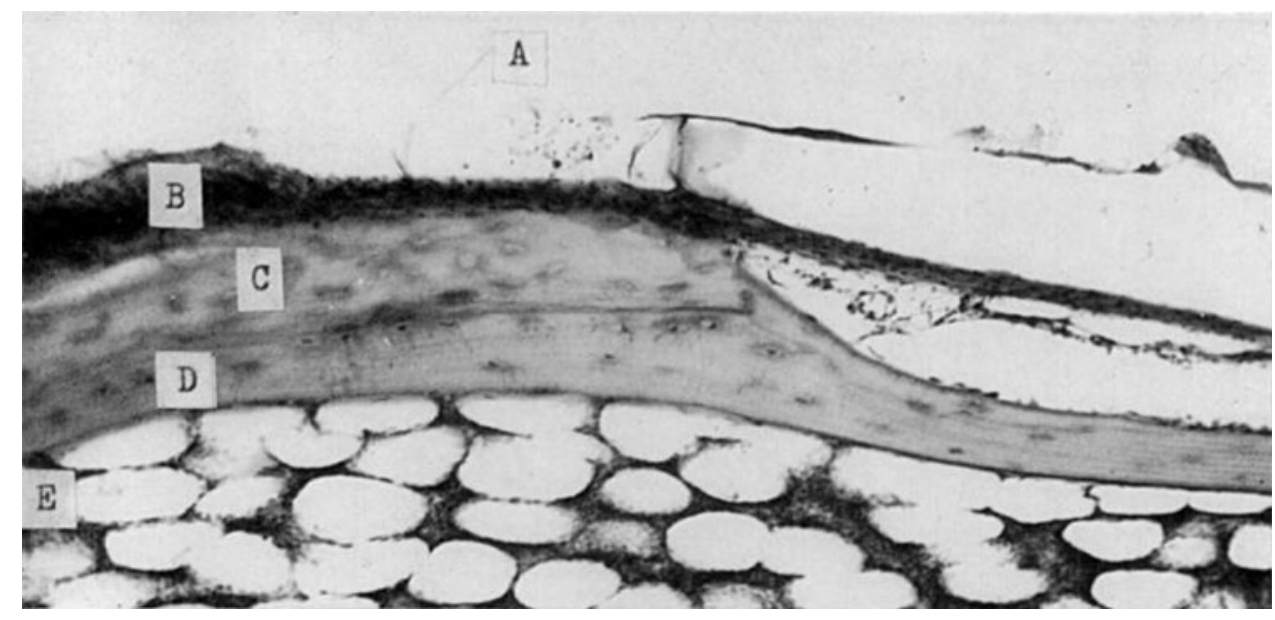

Histology of the cement-bone bond in all six cases showed a uniform appearance. In no case was there sclerosis of cancellous bone. Cancellous bone spaces, which must have been broken into at operation, had repaired themselves and sealed off the open end of the trabecular space with a thin layer of lamellar bone. In a few cases there were superficial lamellae of bone with the spaces empty of osteocytes, but on the side away from the cement there was always a thin layer of new lamellar bone. A striking feature was the normal appearance of the cellular contents of the marrow spaces in the layer of spongiosa in contact with the cement (Fig. 8). There was no fibrosis which might have been expected from the presence of a foreign body giving rise to mechanical or chemical irritation.

In no case was there any evidence of abnormality of the periosteal tissue on the surface of the specimen. As in normal adult bone the periosteum was so thin as to be almost indistinguishable by the naked eye.

\section{Direct Examination by Re-exploration}

Of the 455 cases in which femoral head prostheses have been cemented into the femoral shaft forty-three hips in forty-one patients have been re-explored more than six months after operation and the state of fixity of the prosthesis has been tested. If the five patients are added in whom direct examination of the fixation of the prosthesis was made at necropsy (the sixth being one of those re-explored), there are forty-eight hips for analysis. Of these, four were for prosthetic replacement of the femoral head alone, while the remaining forty-four were prosthetic femoral head replacements combined with a Teflon acetabular prosthesis (Charnley 1961). Five of the re-explorations were for infection and the sixth had the radiological appearances of infection though no pus was found. Of these only one had a loose prosthesis. It is, therefore, unnecessary to make a separate statistical analysis for looseness related to infection. It was evident that the infection had started in the cavity of the arthroplasty and slowly tracked into the cement-bone bond. Only when the infection had been present for more than a year was there evidence that infection had reached the distal half of the prosthesis.

Only two cases out of forty-eight hips showed looseness of the prosthesis. These loose prostheses were explored and removed three years after insertion but there was no doubt that they had both been loose as soon as one year after operation. The two cases explored four years after insertion were both rigidly fixed in position. Table 1 shows the cases re-explored in relation to the time after operation. The peak incidence of re-exploration was between two and two and a half years which corresponds with the usual time for the wearing out of the soft Teflon socket as it was used in 1960 and 1961 .

\section{Radiological Appearances of Cases Re-explored}

The five cases examined at post-mortem (the sixth appears in the re-exploration figures) have been added to the fortythree hips examined at re-exploration, giving forty-eight hips for study.

\section{Absorption of the Femoral Neck}

An almost constant feature was the absorption of bone in the stump of the femoral neck next to the collar of the prosthesis, a process which is complete one and a half years after operation. Radiographically the collar of the prosthesis projected above the level of the bone sometimes by as much as one and a half inches (Figs. 9 to 11). This appearance results from the fact that, if the stump of the calcar femorale absorbs, the cement will prevent the prosthesis from sliding into the medullary canal. The two cases with loosening of the prosthesis have been excluded as the absorption was concealed by the downward shift of 
Table 1 Fixation observed at exploration of forty-eight hips

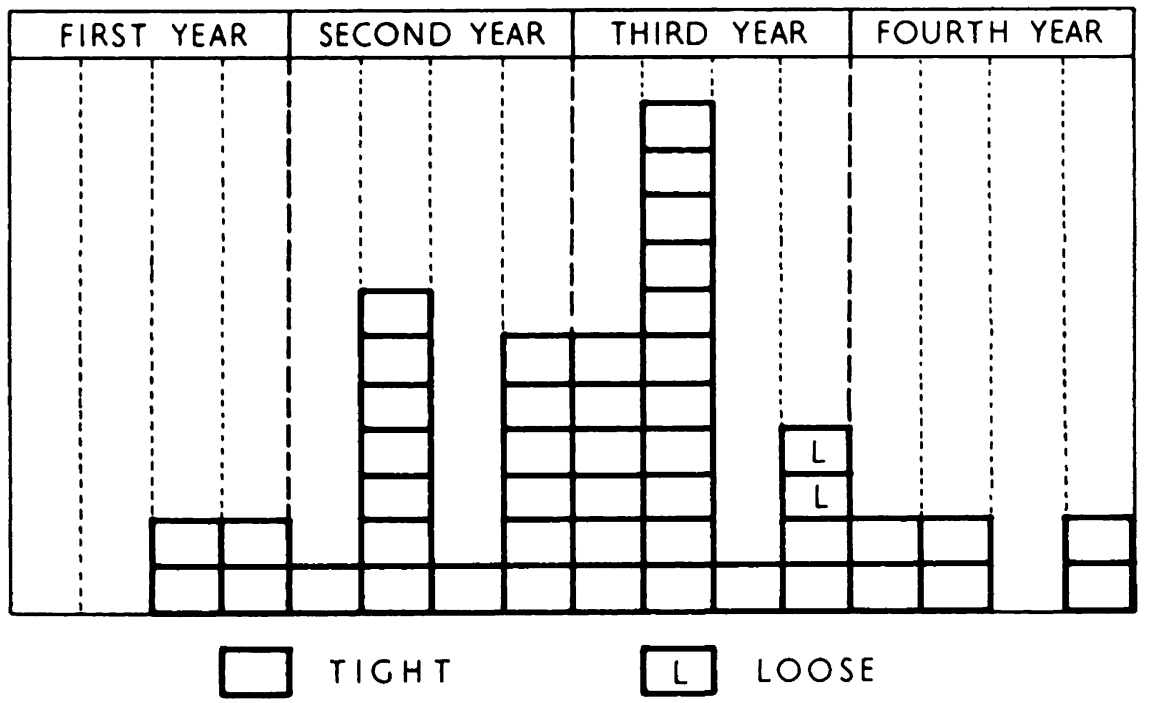

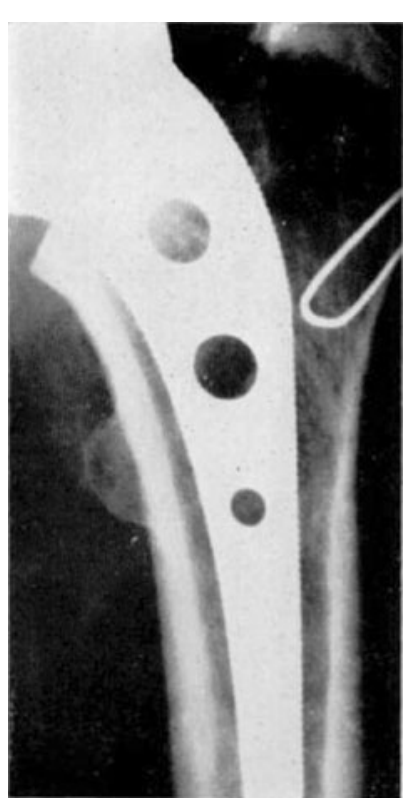

Fig. 9 Absorption of bone at the upper end of the femur. The prosthesis does not settle lower down in the shaft because weight is taken by the cement. Appearances immediately after operation.

the prosthesis. The amount of absorption of the femoral neck in relation to time is shown in Table 2. It is evident that absorption is not directly related to time because there were two cases with absorption of no more than one-eighth of an inch after three and a quarter years and one case with one inch of absorption after one year.

It must be emphasised that this rather alarming appearance does not mean any defect in function. This shows that the cement transmits load directly to the cortex of the shaft of the femur through the distal two-thirds of the

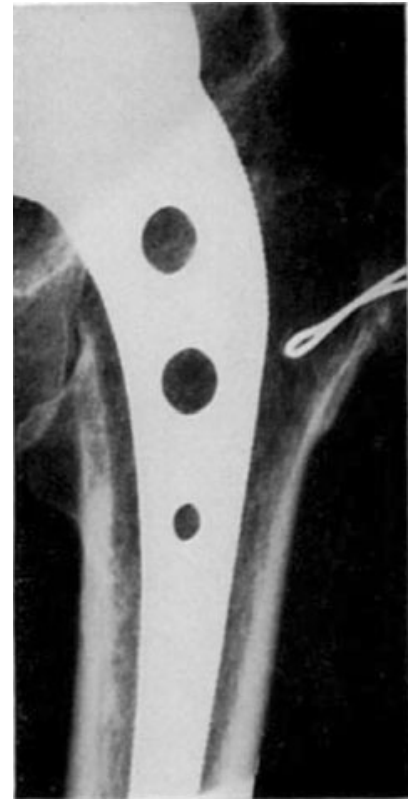

Fig. 10 Absorption of bone at the upper end of the femur. The prosthesis does not settle lower down in the shaft because weight is taken by the cement. One and a half years later.

stem of the prosthesis and that the weight-bearing function of the calcar femorale is by-passed. The absence of function may be one of the reasons for the absorption of the calcar femorale, in accordance with Wolff's Law, but it is more likely that the cause of absorption is destruction of the blood supply. Three factors are responsible for the ischaemia: 1) the "end effect" of interrupting longitudinal circulation in the Haversian canals of the dense cortical bone of the calcar femorale by the section through the neck; 2) the stripping of periosteum from the calcar 
femorale during dislocation of the hip due to tension of the capsular ligaments and forceful surgery; and 3) the curettage of the spongiosa of the medullary canal. It is not surprising that bone should be absorbed which has had both its periosteal and its endosteal blood supply removed, and at the same time had its remaining vitality threatened by cement in the chemical process of polymerisation.

Since care has been taken not to strip periosteum or to curette the medullary cavity, the amount of absorption has been much reduced. In degenerative and rheumatoid arthritis the periosteal covering of the femoral neck strips

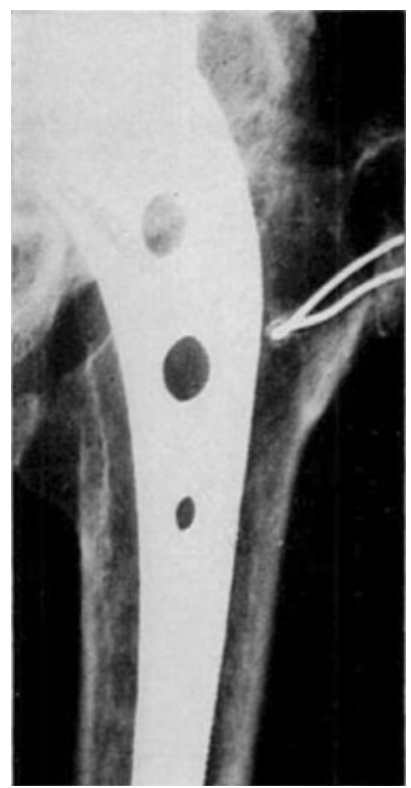

Fig. 11 Absorption of bone at the upper end of the femur. The prosthesis does not settle lower down in the shaft because weight is taken by the cement. Two and a half years after operation and immediately before attempting extraction of the prosthesis. Note that enlargement of the medullary canal as a result of osteoporosis did not indicate looseness of fixation of the cement. very easily, and special care must be taken to divide all tight structures before dislocating the femoral head. In recent fractures of the femoral neck, where the tissues are normal and their adherence to bone not reduced by an arthritic process, absorption of the calcar femorale after cementing a femoral head prosthesis is negligible.

It is probable that the destruction of the blood supply in the calcar femorale is always greater when cement is used than when it is not, but absorption is concealed when cement is not used because the prosthesis can shift down the medullary canal. If cement is not used the amount of absorption of the calcar may not be evident unless the relative levels of the femoral head and the tip of the great trochanter are carefully measured and compared with the post-operative radiographs.

\section{Evidence of Looseness}

It was decided to take the radiographic appearances at the tip of the stem of the prosthesis as a criterion. This criterion was chosen because the appearances in the spongiosa at the intertrochanteric level are often difficult to compare. In none of the 455 cases where cement was used was a radiological appearance seen like that illustrated in Figs. 12 and 13 of an uncemented prosthesis which was proved to be loose when re-explored after one and a half years of weight bearing.

\section{Porosis of the Femoral Shaft}

As the maintenance of bone density in the cortical bone of the femoral shaft is a sign that normal weight transmission is taking place, it is important to study this feature when cement has been in position for some years. Unfortunately differing exposures of radiographs render comparisons of

Table 2 Absorption of the femoral neck in inches

\begin{tabular}{|c|c|c|c|c|c|c|c|}
\hline FIRST & YEAR & SECON & YEAR & THIRD & YEAR & FOURTH & YEAR \\
\hline $\begin{array}{l}3 \\
\vdots \\
\vdots \\
\vdots \\
\vdots \\
\vdots \\
\vdots \\
\vdots \\
\vdots \\
\vdots\end{array}$ & $\vdots$ & $\begin{array}{r:}0 \\
0 \\
0 \\
0\end{array}$ & 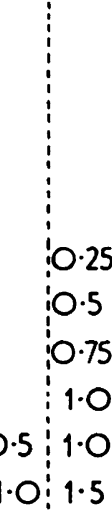 & $\begin{array}{c:c} & 0.25 \\
& 0.5 \\
& 0.5 \\
& 0.5 \\
& 0.5 \\
0 & 0.5 \\
0.5 & 1.0 \\
0.5 & 1.0 \\
0.5 & 1.0 \\
1.0 & 1.0 \\
1.5 & 1.0\end{array}$ & $\begin{array}{r:r:r} & 0.75 \\
& 0.75 & 1.0\end{array}$ & 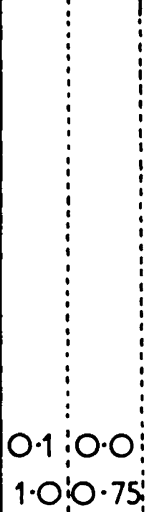 & $\begin{array}{c}0.1 \\
0.5 \\
\vdots \\
\\
0 \\
0\end{array}$ \\
\hline
\end{tabular}


little value except for gross changes. In many cases osteoporosis was present at the time of operation and it was impossible to be sure whether or not this had remained the same after the operation. It was easy to pick out the films where the cortical bone had retained its normal density and thickness. These were classed as normal and the remainder were classed as osteoporotic.

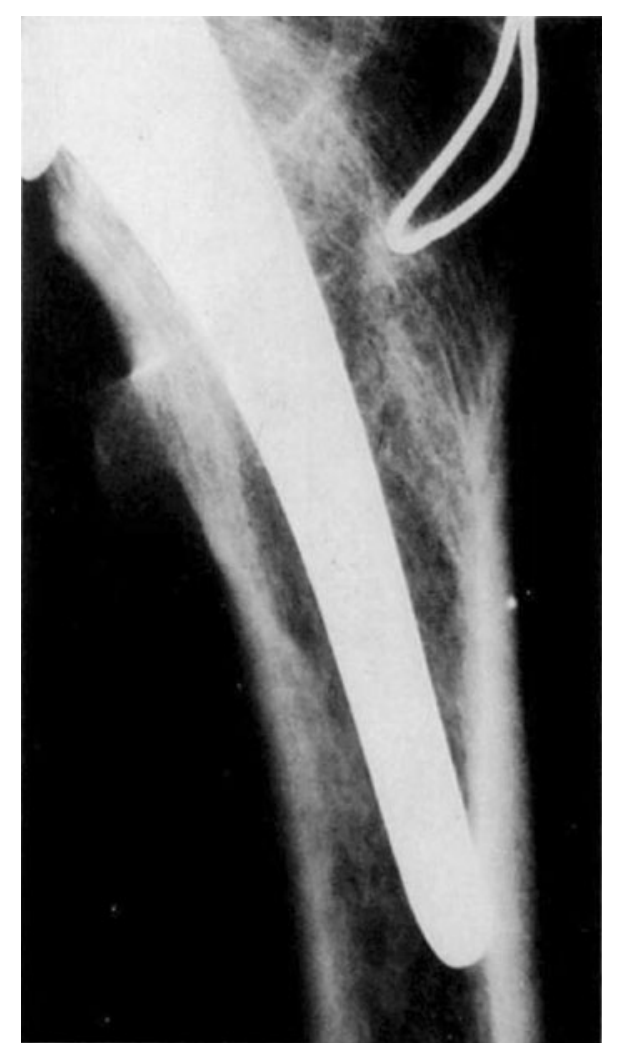

Fig. 12 Post-operative radiological appearance of bone in a case where an uncemented prosthesis was proved to be loose by exploratior.
Table 3 shows the distribution of the cases with normal shaft density in relation to the time after operation. The septic cases and the two loose prostheses have been excluded. leaving forty-one cases for study. The incidence of osteoporosis in this Table might appear high but it must be remembered that anything which was not obviously normal has been called osteoporosis. Since all these hips are abnormal it is astonishing to find that any could retain

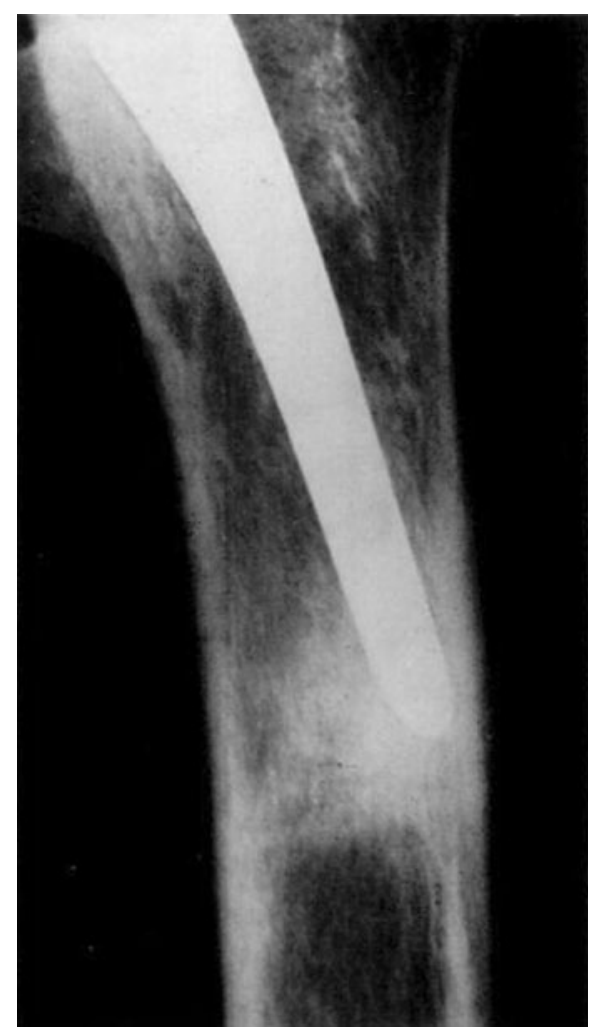

Fig. 13 Radiological appearances of the same case one and a half years later.

Table 3 The incidence of normal bone density of the femoral cortex after operation

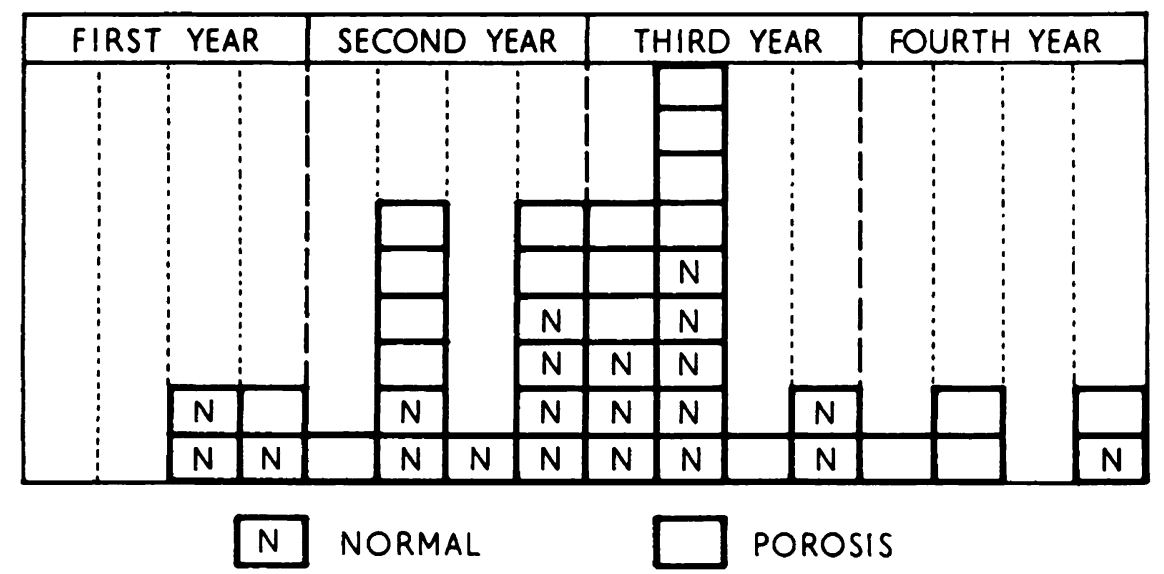


" "normal" bone density for two or three years. The Table shows no significant difference in the proportion of cases with normal bone density in the second year compared with the third year. In the fourth year the proportion of cases with normal bone density declined sharply, but the numbers are too few for comparison. One of the patients with osteoporosis at four years was known to have used crutches and to have avoided bearing weight for a great part of this time as a result of a defective and painful arthroplasty, so the osteoporosis could have been due to simple disuse rather than any harmful local effect of the cement.

We can say therefore that at least for three years there is no evidence of loosening of the cemented prosthesis as a result of any process related to the passage of time and thus to continuous noxious influence from the cement.

The small series of forty-eight cases, in which the state of fixation of the prosthesis was checked directly by re-exploration, is obviously of greater importance than a broad clinical impression gained from the larger series of approximately 410 cases where no exploration was needed. Nevertheless the impressions from the larger group are of value in emphasising that the deductions from the smaller group are not exceptional and are likely to be typical. The radiographic appearances of the cement-bone bond in the larger series confirms the findings in the smaller series that there is no bone reaction in the vicinity of the cement, and that some absorption of the cut surface of the femoral neck is a constant finding and devoid of clinical significance. The larger series shows that sepsis is not significantly higher after the use of cement than after other forms of major surgery on the hip using permanent implants. Out of the total series of 455 hip operations there has been an infection rate of 4.4 per cent and in the last 176 cases after

Fig. 14 Showing how extraction was possible only by fracturing the cement to leave behind the cement distal to the fenestrations in the shaft of the prosthesis. taking special precautions in the theatre the infection rate has been less than 1 per cent. The larger series does not suggest that there are obscure complications in the soft tissues which might be attributed to the effects of chemicals or allergy to the cement.

\section{Strength of Fixation}

Some idea of the strength of the cement fixation may be gained from a description of the difficulties encountered in extracting these prostheses. When the prosthesis had a solid stem of the Thompson type, it was easy to knock out the prosthesis leaving the cement behind since adhesion between the cement and surface of the metal does not occur. When the prosthesis was of the Moore type, with a fenestrated stem, extraction was not possible without gross mutilation of the bone and even fracture of the shaft of the femur. By using a punch or an engineer's cold chisel lodged under the collar of the Moore prosthesis, it was sometimes possible to knock it out by repeated hammer blows using great violence. It was evident that the most powerful grip of cement to bone lay in the distal part of the shaft and not in the trochanteric region, because the prostheses came out as a result of the cement fracturing at the level of the distal fenestration. The proximal cement came out with the prosthesis but the distal cement was left behind. Some idea of the violence needed to extract these prostheses can be gained from the damage to the stainless steel (Figs. 14 and 15). The strength of the fixation of the

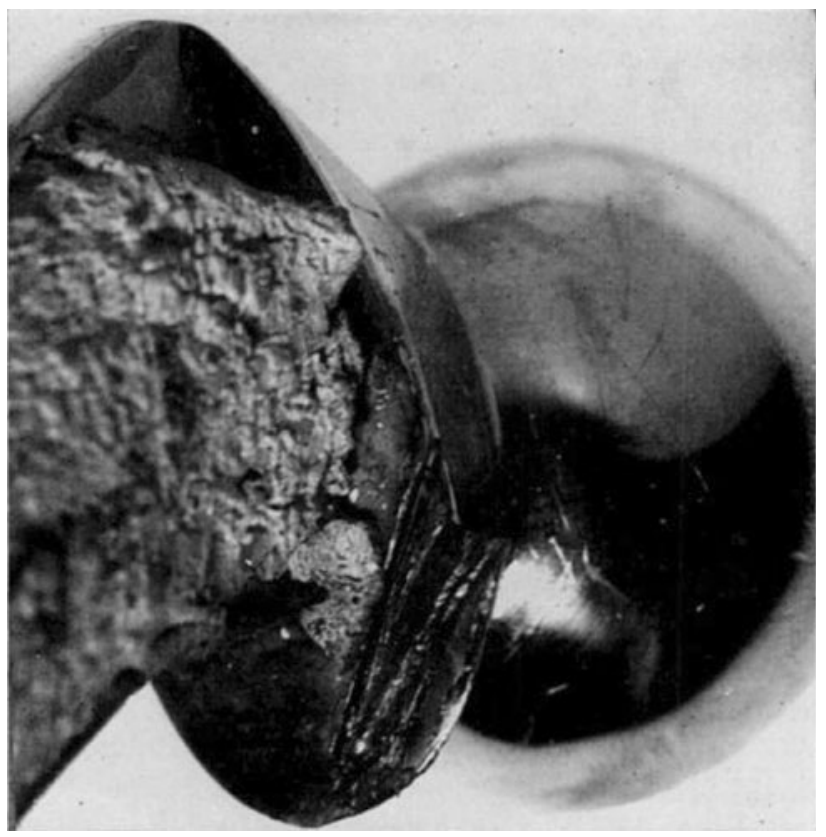

Fig. 15 An Austin Moore prosthesis inserted with cement showing the damage to the metal caused by the violence needed to extract it after one and a half years. 


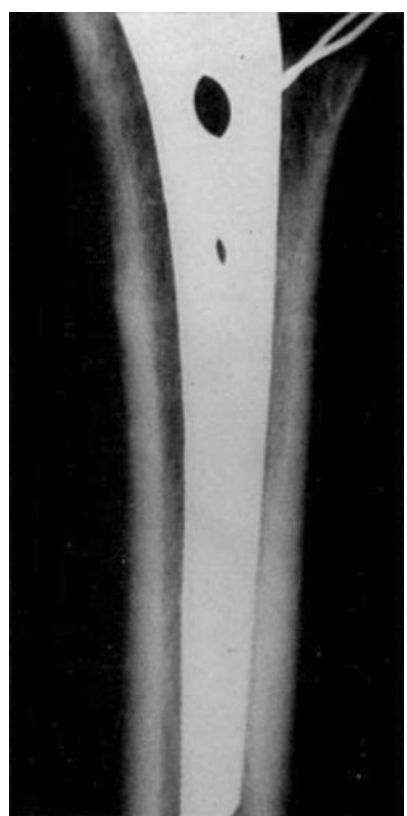

Fig. 16 Same case as Figs. 9, 10 and 11. A prosthesis which defied extraction after two and a half years in situ. The stem of the prosthesis had a serrated surface.

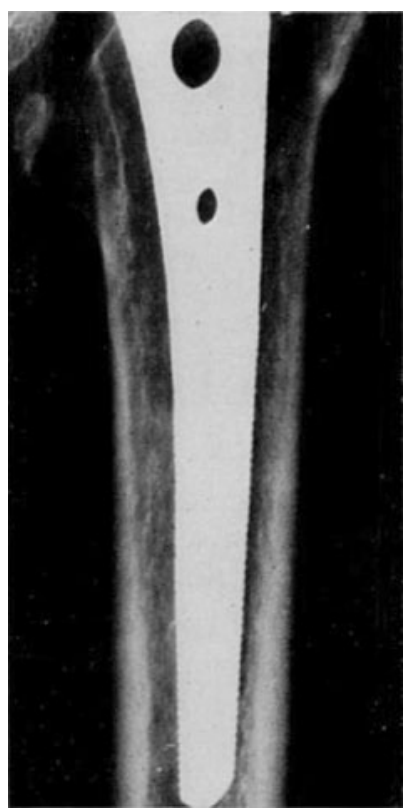

Fig. 17 Same case as Figs. 9, 10 and 11. A prosthesis which defied extraction after two and a half years in situ. Note that enlargement of the medullary canal did not indicate loosening of the attachment of the cement.

cement to bone in the distal half of the prosthesis was demonstrated in one instance where the prosthesis had been specially made with serrations on the surface of the stem for the express purpose of holding the cement. It was decided to extract the prosthesis and convert the hip into a

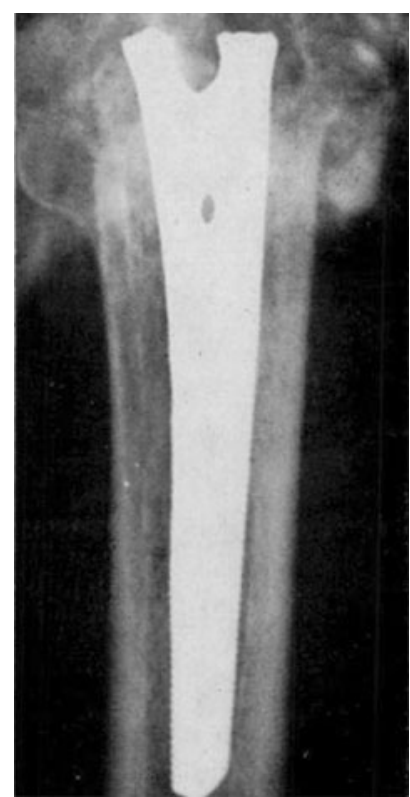

Fig. 18 Same case as Figs. 9, 10 and 11. A prosthesis which defied extraction after two and a half years in situ. After failure to extract the head has been removed. (This indicates that cement is more rigidly anchored in the distal part of the femur than in the proximal part (unlike Figs. 14 and 15, where the distal stem of prosthesis is smooth).)

pseudarthrosis because of mechanical failure of a Teflon socket. The prosthesis, however, defied extraction, and the head had to be cut off, leaving the stem in situ. In this patient the cement-bone junction had been exposed to the load of active weight bearing for two and a half years (Figs. 16 to 18).

\section{Management of the Infected Case}

The problem of infection is obviously very serious when cement has been used to anchor a prosthesis, but extraction of the prosthesis in order to make a Girdlestone's pseudarthrosis is not as formidable as I had originally imagined. If infection occurs the prosthesis should be removed without delay. Primary healing can then be anticipated because the cement delays the spread of infection to the medullary canal of the femur. It is essential that all the cement should be removed if a sinus is to be avoided. The lateral approach to extract the prosthesis and cement is the best, using a T-shaped incision with the transverse limb of the incision in Nelaton's line and the vertical limb in the line of the shaft of the femur. The abductor muscles are elevated from the surface of the greater trochanter by a sharp chisel raising tiny shingles of bone. The hip is then dislocated, the prosthesis knocked out and what cement can be reached from the open end of the medullary canal is chipped out. The lateral surface of the shaft of the femur is 
then exposed for a distance of about five inches and guttered to expose the rest of the cement. The operation is completed by passing a fine suction drain through the guttered femur into the acetabulum and closing the wound. Skin traction is then used for six weeks. Because of the difficulty of removing cemented fenestrated prostheses, the Thompson type of prosthesis is the one most suitable for use with cement.

\section{Discussion}

The first experimental study of the use of cold-curing acrylic cement in orthopaedic surgery was by Wiltse, Hall and Stenehjem (1957), who came to the conclusion that in the rabbit or monkey self-curing methyl methacrylate caused no more tissue reaction than Vitallium. Their work was confined to animals and they did not use cement in humans. Despite encouraging histological appearances after twenty-four months of cement in the medullary canal they still had some reservations on the possible ill-effects of heat generated during the process of polymerisation, and they stated that cement would not remain absolutely tight against the bone.

In 1951 Sven Kiaer (published 1953) described the bonding of plastic prostheses to bone with selfpolymerising plastics, a technique learnt from dental surgeons, and in 1953 Haboush gave a clear account of using it to anchor long-stemmed metal prostheses. Both these attempts confined the acrylic cement to the cancellous bone in the proximal part of the femur. My observations led me to suppose that this technique would fail, even with a longstemmed prosthesis, as the cement bond is not as effective in the cancellous bone of the proximal part of the femur as it is lower down in the medullary canal.

It is easy to propose theoretical objections against acrylic cement, such as allergy to the monomer and illeffects from the heat which accompanies setting. In the literature on soreness of the mouth attributed to dentures made of acrylic, Fisher (1956) considered the liquid monomer as the cause, but Nyquist (1958) and Smith (1959) considered that other factors such as a defective fit of the denture could not be excluded and that the amount of available free monomer was insignificant. It would seem fairly certain that it is only free monomer which can cause allergy and that the amount of monomer which can escape from a polymerised mass containing as much as 5 per cent of residual monomer is very slight. It seems likely that free monomer in the surface layers of the polymerised mass can escape over a limited period, but that the rest remains safely inside the solid mass. Tests by taste and smell indicate that monomer is not escaping continuously from the polymerised resin, and one must not forget that the simple tests of taste and smell are much more sensitive than chemical tests.

Tissue reaction to acrylic resin is more likely to occur when the surface of the cement is continually abraded, because this will release monomer as the deeper layers are exposed. In the tissues next to an acrylic Judet prosthesis there are always abnormal histological changes, but experience of these prostheses in the human body for seven to twelve years shows that these histological changes are not accompanied by clinical pathology in the soft parts. This is quite remarkable when one considers that, after ten or fifteen years, as much as 5 grammes of acrylic material may have been abraded and must have been removed in particles of almost molecular size since the surface retains optical transparency. Particles liberated in such finely divided form would be likely to exert their maximum noxious effect locally or systemically, and this I believe is a very strong argument for believing that methylmethacrylate is harmless in animal tissues.

As a result of heat generated by the reaction of polymerisation, osteocytes in the superficial layers of the cancellous bone are killed by the cement. Wiltse $e t$ al. found temperatures as high as $\mathbf{1 7 5}$ degrees Fahrenheit in a lump of plastic 0.75 inch thick, but in cement no thicker than 0.25 inch he found the rise of temperature no higher than 15 degrees Fahrenheit. I have never observed any change in the colour of blood in contact with this cement at the moment of setting, which suggests that if the osteocytes are killed the proteins of the bone will probably not be denatured.

The success of the cement technique probably depends on a smooth transition, without the intervention of a loose phase, from a bond with dead bone to a permanent bond through a thin layer of dense fibrous tissue. The distribution of the load of the body over a large area reduces local stresses on the fibrous intersection to such a low level that a thin layer of dense fibrous tissue can transmit load as efficiently as bone. Acrylic cement does not adhere to bone like glue; it merely forms an accurate cast of the interior of the bone so that load is transmitted evenly over all parts of the interface between cement and cancellous bone.

\section{Summary and Conclusions}

1. The use of acrylic cement in bonding femoral head prostheses to bone is described.

2. No sign of deterioration of the bond between the cement and bone has been seen in histological preparations up to three and a quarter years after operation, and no harmful effects have been recognised, or suspected, in 455 patients in whom it has been used. 
3. The technique is considered justifiable in elderly patients where the medullary canal is large and the cortex of the femur is thin and brittle.

\section{References}

Charnley, J. (1960): Anchorage of the Femoral Head Prosthesis to the Shaft of the Femur. Journal of Bone and Joint Surgery, 42-B, 28.

Charnley, J. (1961): Arthroplasty of the Hip. A New Operation. Lancet, $\mathbf{i}, 1,129$.

Fisher, A. A. (1956): Allergic Sensitization of the Skin and Oral Mucosa to Acrylic Resin Denture Materials. Journal of Prosthetic Dentistry, 6, 593.
Haвoush, E. J. (1953): A New Operation for Arthroplasty of the Hip. Bulletin of the Hospital for Joint Diseases, 14, 242.

KIAER, Sven (1953): Experimental Investigation of the Tissue Reaction to Acrylic Plastics. Cinquième Congrès international de Chirurgie orthopédique, Stockholm 1951. Bruxelles: Imprimerie Lielens.

Nyquist, G. (1958): Sensitivity to Methyl Methacrylate. Transactions of the Royal Schools of Dentistry. Stockholm and Umea: No. 1, Series $2: 1,35$.

Sмiтh, D. C. (1959): The Acrylic Denture Base; Some Effects of Residual Monomer and Peroxide. British Dental Journal, 106, 331.

Wiltse, L. L., Hall, R. H., and StenehJem, J. C. (1957): Experimental Studies Regarding the Possible Use of Self-Curing Acrylic in Orthopaedic Surgery. Journal of Bone and Joint Surgery, 39-A, 961. 